嘼 


\title{
Reference Tissue Models for FDG-PET Data: Identifiability and Solvability
}

\author{
Mara Scussolini, Sara Garbarino, Michele Piana, Gianmario Sambuceti, and Giacomo Caviglia
}

\begin{abstract}
A reference tissue model (RTM) is a compartmental approach to the estimation of the kinetic parameters of the tracer flow in a given two-compartment target tissue (TT) without explicit knowledge of the time activity curve (TAC) of tracer concentration in the arterial blood. An "indirect" measure of arterial concentration is provided by the TAC of a suitably chosen one-compartment reference tissue (RT). The RTM is formed by the RT and the TT. In this paper, it is shown that the RTM is identifiable, i.e., the rate constants are uniquely retrievable, provided that a selection criterion for one of the coefficients, which is based on the Logan plot of the RT, is introduced. The exchange coefficients are then evaluated by the application of a Gauss-Newton method, with a regularizing term, accounting for the ill-posedness of the problem. The reliability of the method is validated against synthetic data generated according to realistic conditions, and compared with the full two-compartment model for the TT, here used as "gold standard". Finally, the RTM is applied to the estimate of the rate constants in the case of animal models with murine cancer cell lines CT26 inoculated.
\end{abstract}

\section{INTRODUCTION}

$\mathbf{P}$ OSITRON emission tomography with 2 -deoxy-2- ${ }^{18} \mathrm{~F}$ fluoro-D-glucose (FDG-PET) is a widely used tool for in vivo reconstruction of the tissue glucose metabolism, because the process of flow or accumulation of tracer in a target tissue is regarded to resemble rather closely the behaviour of glucose [19], [24].

Following a model originated in the framework of pharmacokinetics, the processing of FDG-PET data is based on compartmental analysis. The unknown tracer concentrations of the compartments of the target tissue (TT) satisfy a linear non-homogeneous system of ordinary differential equations (ODEs), with constant coefficients, expressing the general principle of tracer conservation [11], [19], [24]. The tracer concentrations are the state variables; the constant coefficients represent the rate constants (or rate coefficients) of the tracer flow between compartments; the input function (IF), or source term, is proportional to the time activity curve (TAC) of the tracer concentration in the arterial blood; the proportionality

M. Scussolini is with the Dipartimento di Matematica, Università di Genova, Genova, Italy.

S. Garbarino is with the Centre for Medical Image Computing, Department of Computer Science, University College London, U.K. (s.garbarinoducl.ac.uk).

M. Piana is with the Dipartimento di Matematica, Università di Genova, and CNR-SPIN, Genova, Italy.

G. Sambuceti is with the Dipartimento di Medicina Nucleare, IRCCS-IST San Martino, and Dipartimento di Scienze della Salute, Università di Genova, Genova, Italy.

G. Caviglia is with the Dipartimento di Matematica, Università di Genova, Genova, Italy. coefficient is a further unknown rate constant. In general, compartmental analysis aims at the determination of the unknown rate coefficients from the TACs of arterial blood and TT, which in turn are recovered by drawing regions of interest (ROIs) on the reconstructed images of activity distribution. Mathematically speaking, compartmental analysis deals with the inverse problem of determining the coefficients of the system of ODEs given dynamic PET data on TT and IF.

In principle the IF can be determined by measuring the TAC on a ROI positioned over a sufficiently large blood pool, such as the left ventricle. However, the procedure is subject to systematic errors arising from, e.g., partial volume effects, spillover, cardiac motion, and the low resolution of PET cameras (see [25] and the related references). It is also well known that at the very beginning of the diffusion process the arterial TAC shows a very high peak, which is difficult to estimate reliably. As a consequence, the estimate of the values of the rate coefficients is subject to systematic errors.

To avoid direct reference to the IF, alternative approaches have been developed which have been referred to as reference tissue models (RTMs). The idea is that a TAC measured over a ROI belonging to a suitably chosen reference tissue (RT) may be used as an "indirect" IF for the TT [19]. Reference tissue approaches are reported to provide robust estimates of the unknown parameters if the RT can be modeled as a one-compartment system, e.g., in the case of a radiotracer with reasonably fast kinetics or negligible specific binding [8], [15], [19], [20], [26], [27]. Rather similar approaches (dualtracer models) have also been developed such that, in place of considering the RT, a second untargeted tracer is injected and diffused inside the TT [20]-[23].

A further advantage associated with the use of RTMs, with respect to semi-quantitative methods such as SUV or SUVr measurements, depends on the fact that the distribution of FDG at PET examinations is heavily influenced by renal and liver absorption, pathological conditions, and assumption of drugs [6], [7], [16]. While these effects might modify the diagnostic interpretation of PET data, comparison of the activities in two different (possibly nearby) tissues is likely to overcome these distortion mechanisms.

The procedure described in the present paper may be regarded as a revisitation of reference tissue approaches. We consider an RTM which comprises a two-compartment TT and a one-compartment RT. The TT contains both free and bound tracer, while the RT is supposed to be free of tracer receptors. We assume that the TACs of the TT and the RT are reconstructed from image data. No a priori restriction on rate coefficients is introduced; in particular, we do not require any 
equality of volume distributions for TT and RT, a rather strong assumption which has been subject to criticism [20], [21]. In principle the RTM depends on 6 unknown kinetic parameters, which however are reduced to 5 with reference to the value of the slope of the asymptotic Logan plot of the RT [14].

Our approach to the inverse problem for the RTM is driven by the request of identifiability, which means that the kinetic coefficients are requested to be uniquely determined by the data (in a noise-free framework). We show that the RTM with 5 coefficients, and in a general framework, is identifiable. Identifiability does not hold in the case of negligible blood fractions. In that case, the criterion for the selection of the acceptable set of rate constants is provided by comparison of the numerical results coming from the inversion procedure with data from the asymptotic Logan plot of the RT. The computational tool in our paper is a regularized Gauss-Newton scheme [4], [7], which proves to be very efficient and robust. The proposed RTM model together with the procedure for the solution on the inverse problem are validated on synthetic data, compared with the gold standard full kinetic approach (comprising the IF), and applied to a group of four animal models with murine cancer cell lines CT26.

The scheme of the paper is as follows. Section III provides the mathematical setup of the RTM. Section III discusses the identifiability problem for the RTM. Section [V] provides the numerical validation of the computational method for the reduction of the RTM, while Section $\mathrm{V}$ applies the method against experimental datasets. Our conclusions are offered in Section VI

\section{MAthematicAl MOdel}

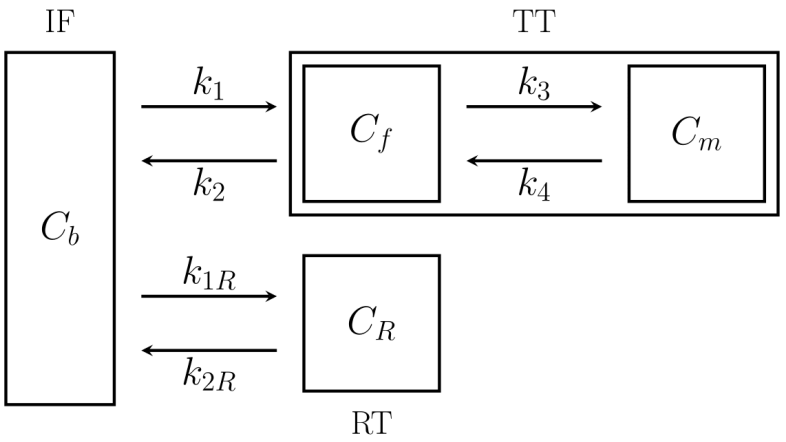

Fig. 1. The reference tissue compartmental model used in this paper.

We assume that the conditions for applicability of compartmental analysis are satisfied [11]. Our aim is to reconstruct the exchange coefficients for tracer kinetics in a given TT. Usually, the input function is given by the arterial blood concentration $C_{b}$ but, by the introduction of an additional RT, we avoid complete determination of the TAC for $C_{b}$.

The PET data considered in the evaluation of the exchange coefficients are the TACs of the TT and the RT. Although the concentration $C_{b}$ is formally introduced in order to describe the tracer kinetics in the TT and RT, only the asymptotic values of $C_{b}$ are involved in the solution of the inverse problem for tracer coefficients.

\section{A. Compartmental schemes}

This subsection is devoted to the description of the compartmental models adopted to characterize kinetic processes of radio-tracer in RT and TT. The RT and TT are represented as one-compartment and two-compartment models, respectively, and the simultaneous interpretation of the two models leads to the mathematical scheme of the RTM.

1) One-compartment reference tissue: The concentration of bound tracer in the RT is taken as significantly low to be neglected [8], [15], [19], [20], [26], [27]. Thus the RT is modelled as a single compartment of tracer concentration $C_{R}$. As a consequence of the balance equation for tracer it is found that $C_{R}$ solves the Cauchy problem

$$
\dot{C}_{R}=-k_{2 R} C_{R}+k_{1 R} C_{b}, \quad C_{R}(0)=0,
$$

where $k_{1 R}$ and $k_{2 R}\left(\min ^{-1}\right)$ are the rate coefficients for tracer exchange from arterial blood to reference tissue, and conversely. The arterial blood concentration $C_{b}$ is the input function.

We assume that the volume fraction occupied by blood in RT can be estimated or measured a priori, and is denoted with $V_{b R}$. Thus the total radioactivity concentration is given by

$$
\mathscr{C}_{R}=\left(1-V_{b R}\right) C_{R}+V_{b R} C_{b}
$$

and is measured by drawing ROIs on the overall tissue selected to work as a reference. Henceforth $\mathscr{C}_{R}$ is regarded as a datum.

We let

$$
k_{2 R}=\lambda k_{1 R} .
$$

The interpretation of the constant adimensional parameter $\lambda$ follows from the observation that

$$
\lambda=\frac{k_{2 R}}{k_{1 R}}=\frac{1}{D V_{R T}},
$$

where $D V_{R T}$ is the distribution volume [26] of the RT.

It is found from (1), (2) and the definition of $\lambda$ that

$$
\int_{t_{0}}^{t} \mathscr{C}_{R}=\beta_{1} \int_{t_{0}}^{t} C_{b}+\frac{\left(1-V_{b R}\right)}{k_{2 R}}\left(C_{R}\left(t_{0}\right)-C_{R}\right),
$$

where

$$
\beta_{1}=\left(1-V_{b R}\right) \frac{1}{\lambda}+V_{b R}
$$

and $t_{0}<t$. Division by $\mathscr{C}_{R}$ leads to the equation

$$
Y=\beta_{1} X+\beta_{2},
$$

where

$X=\frac{\int_{t_{0}}^{t} C_{b}}{\mathscr{C}_{R}}, Y=\frac{\int_{t_{0}}^{t} \mathscr{C}_{R}}{\mathscr{C}_{R}}, \beta_{2}=\frac{\left(1-V_{b R}\right)}{k_{2 R}}\left(\frac{C_{R}\left(t_{0}\right)-C_{R}(t)}{\mathscr{C}_{R}}\right)$.

In the plane referred to Cartesian axes $(X, Y)$ equation (6) represents a curve parameterized by $t$. If we assume that $t_{0}$ is sufficiently large that $\mathscr{C}_{R}\left(t_{0}\right)$ is close to the asymptotic equilibrium value then the curve (6) is well approximated by a line of slope $\beta_{1}$. Therefore we can determine $\beta_{1}$ by means of a polynomial curve (of degree one) fitting procedure on (6), utilizing the measured asymptotic values of $\mathscr{C}_{R}$ and $C_{b}$. From the knowledge of $\beta_{1}$ and $V_{b R}$, through equation 
(5) is straightforward to obtain $\lambda$ : therefore, from now on the parameter $\lambda$ is regarded as known.

Since the concentration $\mathscr{C}_{R}$ can be measured with more accuracy than $C_{b}$, in the following analysis we replace $C_{b}$ with its expression in terms of $\mathscr{C}_{R}$, which follows from

$$
\dot{C}_{b}=-\gamma k_{1 R} C_{b}+\frac{1}{V_{b R}}\left(\dot{\mathscr{C}}_{R}+\lambda k_{1 R} \mathscr{C}_{R}\right),
$$

where

$$
\gamma=\left(V_{b R}^{-1}-1\right)+\lambda
$$

Now, this is a differential equation in $C_{b}$, whose solution (when $C_{b}(0)=0$ ) is

$$
C_{b}=\frac{1}{V_{b R}} \int_{0}^{t} e^{-\gamma k_{1 R}(t-\tau)}\left(\dot{\mathscr{C}}_{R}+\lambda k_{1 R} \mathscr{C}_{R}\right) d \tau
$$

also expressed as

$$
C_{b}=\frac{1}{V_{b R}}\left(\mathscr{C}_{R}-\left(V_{b R}^{-1}-1\right) k_{1 R} \int_{0}^{t} e^{-\gamma k_{1 R}(t-\tau)} \mathscr{C}_{R} d \tau\right) .
$$

Here $k_{1 R}$ is regarded as a free parameter. By (10), we will get rid of explicit reference to the TAC of the input function $C_{b}$ in the analysis of the TT.

2) Two-compartment target tissue: With reference to Fig. 1. the TT consists of a compartment of tracer concentration $C_{f}$, accounting for free tracer, and a compartment of concentration $C_{m}$, accounting for metabolized tracer. The state variables $C_{f}$ and $C_{m}$ satisfy the Cauchy problem

$$
\dot{\mathbf{C}}=\mathbf{A ~ C}+k_{1} \mathbf{e}_{1} C_{b}, \quad \mathbf{C}(0)=0,
$$

where

$$
\mathbf{C}=\left(\begin{array}{l}
C_{f} \\
C_{m}
\end{array}\right), \mathbf{A}=\left(\begin{array}{cc}
-\left(k_{2}+k_{3}\right) & k_{4} \\
k_{3} & -k_{4}
\end{array}\right), \mathbf{e}_{1}=\left(\begin{array}{l}
1 \\
0
\end{array}\right) .
$$

The rate constants $k_{1}, k_{2}\left(\mathrm{~min}^{-1}\right)$ describe transfer from arterial blood to free tracer, and conversely; similarly, $k_{3}$ and $k_{4}\left(\mathrm{~min}^{-1}\right)$ refer to transfer from free to metabolized tracer, and conversely.

3) Reference tissue model: The previous models of RT and TT are put together to define the RTM, whose related differential equations are applied in order to determine the rate coefficients from image data.

Replacement of $C_{b}$ by its expression in terms of $\mathscr{C}_{R}$ in equation (11) gives

$$
\begin{aligned}
\dot{\mathbf{C}}= & \mathbf{A} \mathbf{C}+\frac{k_{1} \mathbf{e}_{1}}{V_{b R}}\left(\mathscr{C}_{R}\right. \\
& \left.-\left(V_{b R}^{-1}-1\right) k_{1 R} \int_{0}^{t} e^{-\gamma k_{1 R}(t-\tau)} \mathscr{C}_{R} d \tau\right) .
\end{aligned}
$$

Equation (13) shows the connection between the state variables, their time-derivatives, and the rate constants of TT and RT. It may be regarded as the differential formulation of the mathematical model for the RTM.
Consider the differential equation (13), with the initial condition $\mathbf{C}(0)=0$. The solution $\mathbf{C}$ in terms of $\mathscr{C}_{R}$ is given by

$$
\begin{aligned}
\mathbf{C}= & \frac{k_{1}}{V_{b R}} \int_{0}^{t} e^{\mathbf{A}(\mathbf{t}-\tau)} \mathbf{e}_{1}\left(\mathscr{C}_{R}\right. \\
& \left.-\left(V_{b R}^{-1}-1\right) k_{1 R} \int_{0}^{\tau} e^{-\gamma k_{1 R}(\tau-\sigma)} \mathscr{C}_{R} d \sigma\right) d \tau .
\end{aligned}
$$

Equation (14) is regarded as the integral form of the mathematical model for the RTM.

In principle, the RT and the TT involve 6 rate coefficients, but the condition that $\lambda$ is known reduces the total number of parameters to 5. All of them enter equations (13) and (14), characterizing the RTM. A simplified formulation of the RTM is obtained under the assumption that $k_{3}=k_{4}=0$, which means that the TT contributes only to 2 rate constants, instead of the 4 considered here. This case is known as simplified reference tissue model (SRTM, [15]).

We also remark that in a number of approaches to reference tissue modelling [19], [26], [27] the total number of the unknown parameters for the RTM is reduced by the assumption that the distribution volumes of tracer of the two tissues are equal. In our notations, this means that

$$
\frac{k_{1}}{k_{2}}=\frac{k_{1 R}}{k_{2 R}},
$$

in the simplest cases. Due to recent criticism on this assumption [20], [23] especially in the framework of tumor modelling, we have simply considered the ratio $k_{1 R} / k_{2 R}=D V_{R T}$ as experimentally determined by the Logan plot of the RT. An alternative approach [5] has been recently introduced, and relies on estimates of combinations of parameters based on DCE-MRI data. The present paper has been concerned only with PET data.

\section{B. Measurements modelling}

Equation (14) is the starting point for the compartmental inverse problem of finding the exchange coefficients $k_{1 R}, k_{1}$, $k_{2}, k_{3}$, and $k_{4}$ when the total concentrations of the reference and target tissue are known.

As already observed, the total radioactivity concentration $\mathscr{C}_{R}$ in RT is measured by drawing ROIs on the overall tissue selected to work as a reference.

The total concentration $\mathscr{C}_{T}$ in TT is measured similarly, by drawing ROIs on the overall organ or tissue of interest. If the TT is modelled with a two-compartment compartmental scheme, and the fraction of blood volume is denoted as $V_{b T}$, the total radioactivity concentration in TT, say $\mathscr{C}_{T}$, can be written as

$$
\mathscr{C}_{T}=\left(1-V_{b T}\right)\left(C_{f}+C_{m}\right)+V_{b T} C_{b}
$$

By defining a vector

$$
\boldsymbol{\alpha}=\left(\begin{array}{l}
1-V_{b T} \\
1-V_{b T}
\end{array}\right)
$$

(16) can be thus re-written as

$$
\mathscr{C}_{T}-\boldsymbol{\alpha} \cdot \mathbf{C}-V_{b T} C_{b}=0,
$$


where $\mathbf{C}$ is represented as in (14) and $C_{b}$ as in (10).

In an explicit form, (18) becomes

$$
\begin{aligned}
V_{b R} \mathscr{C}_{T}- & V_{b T} \mathscr{C}_{R}-\boldsymbol{\alpha} k_{1} \int_{0}^{t} e^{\mathbf{A}(\mathbf{t}-\tau)} \mathbf{e}_{1}\left(\mathscr{C}_{R}\right. \\
& \left.-\left(V_{b R}^{-1}-1\right) k_{1 R} \int_{0}^{\tau} e^{-\gamma k_{1 R}(\tau-\sigma)} \mathscr{C}_{R} d \sigma\right) d \tau \\
& +V_{b T}\left(V_{b R}^{-1}-1\right) k_{1 R} \int_{0}^{t} e^{-\gamma k_{1 R}(t-\tau)} \mathscr{C}_{R} d \tau=0
\end{aligned}
$$

Equation (19) is regarded as the RTM equation, where $\mathscr{C}_{T}$ and $\mathscr{C}_{R}$ are measured, the blood fractions $V_{b T}$ ans $V_{b R}$ are supposed to be known, and the kinetic coefficients are free.

\section{IDENTIFIABILITY ISSUES}

Before proceeding to the numerical evaluation of the rate coefficients, we need to discuss the formal identifiability of the problem, namely whether the rate coefficients are uniquely determined by the given input data, under the assumption that they are not contaminated by noise. Accordingly, uniqueness plays the role of an a priori test on the mathematical model proposed for the description of the flow of tracer, which can be performed independently of the numerical values of the data. It is shown in this section that uniqueness does hold for the RTM when the rate coefficient $k_{2 R}$ is constrained as $\lambda k_{1 R}$, and $\lambda$ is estimated thanks to equation (5).

The discussion on uniqueness is based on the differential form (11) of the mathematical model for the RTM; identifiability of the RTM corresponds to uniqueness of the vector $\mathbf{k}_{5}=\left(k_{1 R}, k_{1}, k_{2}, k_{3}, k_{4}\right)$ on $\mathbb{R}_{+}^{5 *}$. Following the procedure used in [3], we consider the Laplace transform of the differential equation (11) in order to reduce our identifiability problem to the identification of the coefficients of a rational fraction.

Denote by $\tilde{f}$ the Laplace transform of any function $f$. Under suitable assumptions of regularity, the Laplace transform of system 11 takes the form

$$
(s \mathbf{I}-\mathbf{A}) \tilde{\mathbf{C}}=k_{1} \mathbf{e}_{1} \tilde{C}_{b},
$$

where

$$
\tilde{C}_{b}=\frac{1}{V_{b R}} \frac{s+\lambda k_{1 R}}{s+\gamma k_{1 R}} \tilde{\mathscr{C}}_{R}
$$

thanks to $(8)$, and where $\mathbf{I}$ is the identity matrix of order 2. Left multiplication by the inverse of $(s \mathbf{I}-\mathbf{A})$ provides $\tilde{\mathbf{C}}$. Replacing $\tilde{\mathbf{C}}$ and $\tilde{C}_{b}$ into the Laplace transform of 18 , which relates the measured data to the solution $\mathbf{C}$, gives

$$
\tilde{\mathscr{C}}_{T}=\frac{V_{b T}}{V_{b R}}\left(\frac{k_{1}}{V_{b T}} \boldsymbol{\alpha}(s \mathbf{I}-\mathbf{A})^{-1} \mathbf{e}_{1}+1\right) \frac{s+\lambda k_{1 R}}{s+\gamma k_{1 R}} \tilde{\mathscr{C}}_{R} .
$$

After substitution of the definitions of $\boldsymbol{\alpha}, \mathbf{e}_{1}$, and $\mathbf{A}$, equation (22) may be expressed in the equivalent form

$$
\frac{V_{b R}}{V_{b T}} \frac{\tilde{\mathscr{C}}_{T}}{\tilde{\mathscr{C}}_{R}}=\frac{Q\left(s, \mathbf{k}_{5}\right)}{P\left(s, \mathbf{k}_{5}\right)}
$$

where $P\left(s ; \mathbf{k}_{5}\right)$ and $Q\left(s ; \mathbf{k}_{5}\right)$ are polynomials in $s$, parameterized by the components of $\mathbf{k}_{5}$, defined by

$$
\begin{aligned}
Q\left(s, \mathbf{k}_{5}\right)= & s^{3}+\left(\bar{k}_{1}+\lambda k_{1 R}+k_{2}+k_{3}+k_{4}\right) s^{2} \\
& +\left(\lambda k_{1 R}\left(\bar{k}_{1}+k_{2}+k_{3}+k_{4}\right)+\bar{k}_{1}\left(k_{3}+k_{4}\right)+k_{2} k_{4}\right) s \\
& +\lambda k_{1 R}\left(\bar{k}_{1}\left(k_{3}+k_{4}\right)+k_{2} k_{4}\right),
\end{aligned}
$$

and

$$
\begin{aligned}
P\left(s, \mathbf{k}_{5}\right)= & s^{3}+\left(\bar{k}_{1 R}+\lambda k_{1 R}+k_{2}+k_{3}+k_{4}\right) s^{2} \\
& +\left(\left(\bar{k}_{1 R}+\lambda k_{1 R}\right)\left(k_{2}+k_{3}+k_{4}\right)+k_{2} k_{4}\right) s \\
& +\left(\bar{k}_{1 R}+\lambda k_{1 R}\right) k_{2} k_{4}
\end{aligned}
$$

where $P$ is also the characteristic polynomial of $\mathbf{A}$, and

$$
\bar{k}_{1}=\left(V_{b T}^{-1}-1\right) k_{1}, \quad \bar{k}_{1 R}=\left(V_{b R}^{-1}-1\right) k_{1 R} .
$$

We recall that $\lambda$ is a fixed, known parameter and we also note explicitly that the left-hand side of 23 is only dependent on measurable (given) data. Suppose $\mathbf{h}_{5}=\left(h_{1 R}, h_{1}, h_{2}, h_{3}, h_{4}\right)$ is an alternative choice of rate coefficients consistent with the data of the problem. This means an equation of the form 23, is necessarily satisfied, which implies that

$$
\frac{V_{b R}}{V_{b T}} \frac{\tilde{\mathscr{C}}_{T}}{\tilde{\mathscr{C}}_{R}}=\frac{Q\left(s, \mathbf{k}_{5}\right)}{P\left(s, \mathbf{k}_{5}\right)}=\frac{Q\left(s, \mathbf{h}_{5}\right)}{P\left(s, \mathbf{h}_{5}\right)} .
$$

The discussion of uniqueness for the RTM is based on 27); specifically, it is shown that 27) does imply that $\mathbf{h}_{5}=\mathbf{k}_{5}$.

Under general conditions, the polynomials $P$ and $Q$ are coprime; therefore the rational fraction $Q / P$ is irreducible. Next we observe that $P\left(s, \mathbf{k}_{5}\right)$ and $P\left(s, \mathbf{h}_{5}\right)$ have the same leading coefficient, and the same condition holds for the polynomials $Q$. This implies that equation (27) is verified if and only if

$$
P\left(s, \mathbf{k}_{5}\right)=P\left(s, \mathbf{h}_{5}\right), \quad Q\left(s, \mathbf{k}_{5}\right)=Q\left(s, \mathbf{h}_{5}\right) .
$$

It follows that equation 27 is equivalent to the following equations

$$
\bar{h}_{1 R}+\lambda h_{1 R}+\phi_{h}=\bar{k}_{1 R}+\lambda k_{1 R}+\phi_{k},
$$

$$
\left(\bar{h}_{1 R}+\lambda h_{1 R}\right) \phi_{h}+h_{2} h_{4}=\left(\bar{k}_{1 R}+\lambda k_{1 R}\right) \phi_{k}+k_{2} k_{4},
$$

$$
\left(\bar{h}_{1 R}+\lambda h_{1 R}\right) h_{2} h_{4}=\left(\bar{k}_{1 R}+\lambda k_{1 R}\right) k_{2} k_{4}
$$

$$
\bar{h}_{1}+\lambda h_{1 R}+\phi_{h}=\bar{k}_{1}+\lambda k_{1 R}+\phi_{k},
$$

$$
\begin{aligned}
& \lambda h_{1 R}\left(\bar{h}_{1}+\phi_{h}\right)+\bar{h}_{1}\left(h_{3}+h_{4}\right)+h_{2} h_{4}= \\
& \quad=\lambda k_{1 R}\left(\bar{k}_{1}+\phi_{k}\right)+\bar{k}_{1}\left(k_{3}+k_{4}\right)+k_{2} k_{4},
\end{aligned}
$$

$\lambda h_{1 R}\left(\bar{h}_{1}\left(h_{3}+h_{4}\right)+h_{2} h_{4}\right)=\lambda k_{1 R}\left(\bar{k}_{1}\left(k_{3}+k_{4}\right)+k_{2} k_{4}\right)$,

for $\mathbf{h}_{5}$ in terms of $\mathbf{k}_{5}$, where $\phi_{h}=h_{2}+h_{3}+h_{4}$ and $\phi_{k}=$ $k_{2}+k_{3}+k_{4}$. 
By comparison with (29), 32, can be replaced by

$$
\bar{h}_{1}-\bar{h}_{1 R}=\bar{k}_{1}-\bar{k}_{1 R} \text {. }
$$

First, let us solve the sub-system 291-31) with respect to $\bar{h}_{1 R}+\lambda h_{1 R}=\gamma h_{1 R}$. We find three solutions:

$$
\begin{aligned}
& \gamma h_{1 R}^{(1)}=\gamma k_{1 R}, \\
& \gamma h_{1 R}^{(2,3)}=\frac{\phi_{k} \pm \sqrt{\phi_{k}^{2}-4 k_{2} k_{4}}}{2} .
\end{aligned}
$$

Solution $h_{1 R}^{(1)}$ leads to $\mathbf{h}_{5}^{(1)}=\mathbf{k}_{5}$. Solutions $h_{1 R}^{(2,3)}$ a priori define two distinct sets of solution. Nevertheless, we can observe that, by replacing (32) in (33) and comparing to (34), we obtain

$$
\begin{aligned}
\left(k_{1 R}-h_{1 R}\right)\left(\left(\lambda h_{1 R}\right)^{2}-\right. & \left(\bar{k}_{1}+\phi_{k}\right) \lambda h_{1 R} \\
& \left.+\bar{k}_{1}\left(k_{3}+k_{4}\right)+k_{2} k_{4}\right)=0 .
\end{aligned}
$$

Therefore, to be solutions of the system (29)-(34), the three solutions defined in 36 have to satisfy 37. For $h_{1 R}^{(1)}=k_{1 R}$, 37) is easily satisfied. For $h_{1 R}^{(2,3)}, 37$ becomes

$$
\left(\lambda h_{1 R}^{(2,3)}\right)^{2}-\left(\bar{k}_{1}+\phi_{k}\right) \lambda h_{1 R}^{(2,3)}+\bar{k}_{1}\left(k_{3}+k_{4}\right)+k_{2} k_{4}=0 .
$$

This equation (once $\bar{k}_{1}$ and $\phi_{k}$ are explicated), represents a constraint on the components of $\mathbf{k}_{5}$, that, in general, is not satisfied. Therefore we can state the following result.

Theorem 3.1: Provided that the components of $\mathbf{k}_{5}$ do not satisfy the constraint $(38)$, the RTM is identifiable.

We observe that in the case in which one of the blood fractions is negligible, we still have uniqueness; the same thing does not happen if both the blood fractions are set to zero. In that case, we can't extend the aforementioned computations and the RTM is not identifiable. Indeed, we have two different solutions:

$$
\mathbf{h}_{5}^{(1)}=\mathbf{k}_{5}
$$

and

$$
\begin{aligned}
\mathbf{h}_{5}^{(2)}=\left(\frac{k_{3}+k_{4}}{\lambda}, \frac{k_{1}}{k_{1 R}} h_{1 R}, k_{2}+k_{3}+k_{4}-\lambda k_{1 R},\right. \\
\left.\lambda k_{1 R}-\frac{k_{2} k_{4}}{h_{2}}, \frac{k_{2} k_{4}}{h_{2}}\right) .
\end{aligned}
$$

In this case, uniqueness is ensured only if the value of $k_{1 R}$ is fixed. Although the kinetic coefficients are not generally identifiable, some macroparameters of clinical interest, such as the total volumes of distribution, are here shown to be identifiable. Indeed, the total volume of distribution for RT is $D V_{R T}=$ $1 / \lambda$, that in our framework is estimated (and therefore is obviously identifiable) by means of the Logan plot. The total volume of distribution for TT is $D V_{T T}(\mathbf{k})=\frac{k_{1}}{k_{2}}\left(1+\frac{k_{3}}{k_{4}}\right)$; if $\mathbf{h}_{5}=\mathbf{h}_{5}^{(1)}$, then clearly $D V_{T T}(\mathbf{h})=D V_{T T}(\mathbf{k})$ holds. If $\mathbf{h}_{5}=\mathbf{h}_{5}^{(2)}$, then

$$
\begin{aligned}
& D V_{T T}(\mathbf{h})=\frac{h_{1}^{(2)}}{h_{2}^{(2)}}\left(1+\frac{h_{3}^{(2)}}{h_{4}^{(2)}}\right) \\
& =\frac{k_{1}\left(k_{3}+k_{4}\right)}{\lambda k_{1 R}\left(k_{2}+k_{3}+k_{4}-\lambda k_{1 R}\right)} \frac{\lambda k_{1 R}\left(k_{2}+k_{3}+k_{4}-\lambda k_{1 R}\right)}{k_{2} k_{4}} \\
& =\frac{k_{1}}{k_{2}}\left(1+\frac{k_{3}}{k_{4}}\right) .
\end{aligned}
$$

Therefore $D V_{T T}$ is identifiable. The distribution volume ratio (with respect to the reference tissue) is also identifiable, being $D V_{T T} / D V_{R T}$.

Finally, we point out three remarks.

Remark 3.2: If we consider the SRTM, corresponding, in our notation, to the case $k_{3}=k_{4}=0$, then the model is identifiable.

Remark 3.3: Identifiability is also ensured for the simplifying assumption (although less simplifying than the SRTM case) of $k_{4}=0$ (i.e. negligible de-phosphorylation).

Remark 3.4: The Logan plot step to estimate $\lambda$ is necessary: indeed, if we consider the model with 6 free parameters (i.e. $k_{2 R}$ is free) then identifiability does not hold.

\section{Numerical Validation with a Gauss-Newton SCHEME}

The 5 tracer coefficients $k_{1 R}, k_{1}, k_{2}, k_{3}$, and $k_{4}$ are estimated by utilizing measurements of the tracer concentration provided by nuclear imaging and applying an optimization scheme.

We re-write equation (18) for the unknown vector parameter $\mathbf{k}=\left(k_{1 R}, k_{1}, k_{2}, k_{3}, k_{4}\right)$ in the form

$$
\mathscr{C}_{T}-\boldsymbol{\alpha} \cdot \mathbf{C}-V_{b T} C_{b}=: \mathcal{F}(t ; \mathbf{k})=0,
$$

where $\mathscr{C}_{T}$ is given, $\mathbf{C}$ is represented as in (14), $C_{b}$ is defined as in (10), and equation (40) is considered at the time points of the observations. Thus, the numerical reduction of the RTM requires the numerical solution of equation 40 by means of an optimization-regularization method. For this purpose we utilize an iterative regularized Gauss-Newton (G-N) method as in [4]. The numerical procedure is first validated by considering synthetic data and then applied to a set of real data.

\section{A. Gauss-Newton scheme}

The present application of the $\mathrm{G}-\mathrm{N}$ approach transforms the non-linear optimization problem of finding the exchange coefficients into a linear problem; this is achieved by computing the Frèchet derivatives of equation 40 with respect to the exchange coefficients, and iteratively increasing the value of the $i$-th approximation of $\mathbf{k}$, say $\mathbf{k}^{(i)}$, to $\mathbf{k}^{(i+1)}=\mathbf{k}^{(i)}+\boldsymbol{\delta}^{(i)}$, where the step-size $\boldsymbol{\delta}^{(i)}$ is determined by equation

$$
\mathbf{F}^{(i)} \boldsymbol{\delta}^{(i)}=Y^{(i)} .
$$

Here the matrix $\mathbf{F}^{(i)}$ encodes the Frèchet derivatives with respect to the exchange coefficients at the $i$-th iteration, and the datum $Y^{(i)}$ is the discrepancy between the measured datum $\mathscr{C}_{T}$ and the predicted datum at the $i$-th iteration $\mathscr{C}_{T}^{(i-1)}$

As in [4] we use a regularized version of equation (41), in order to avoid the ill-posedness of the problem and to take into account the fact that real data are finite in number and affected by noise. Therefore, the equation for the regularized $\mathrm{G}-\mathrm{N}$ scheme is

$$
\left(r \mathbf{I}+\mathbf{F}^{T} \mathbf{F}\right) \boldsymbol{\delta}=\mathbf{F}^{T} Y,
$$

where $r$ is the regularizing parameter which is allowed to change at every iteration. Superscript $(i)$ for the iteration is omitted but implied. 


\section{B. Validation on synthetic data}

In this subsection we describe the performance of our approach to reference tissue compartmental analysis in the case of synthetic data simulated by mimicking the behaviour of a real micro-PET system.

The first simulation is set up in order to test the sensitivity of our model, when the assumption of a priori knowledge of the blood volume fractions is broken. Indeed, in this case, we simulate many data using varying blood fractions (for both RT and TT) and then reconstruct the kinetic coefficients by imposing known blood fractions, different from the one used to generate the data. We show that our model is reliable in retrieving the kinetic coefficients, when the blood fractions are mis-estimated up to a $40 \%$ factor (see Table/I). The results are also compared with a full compartmental model (full-CM) for the TT when $C_{b}$ is known.

Further, we show the sensitivity of the standard RTM when the assumption of equal distribution volumes (15) is broken: the model exhibits a very high sensitivity, failing to reconstruct reliable kinetic coefficients when the assumption is broken (see Table III).

The last simulating experiment is presented to test the reliability of our proposed $\mathrm{G}-\mathrm{N}$ algorithm against the standard Levenberg-Marquardt (L-M) algorithm [1] (see Table III).

In all the scenarios, in order to produce the synthetic data we initially chose realistic ground-truth values for the adimensional parameter $\lambda$ and the tracer kinetic parameters $k_{1 R}, k_{1}, k_{2}, k_{3}, k_{4}$. We chose $V_{b R}=0.025$ and $V_{b T}=0.15$ to be the realistic values for the blood fractions, for the reference tissue and the target tissue respectively (see Real Data Section V for references); in order to test the sensitivity of our model to a non-precise volume fraction estimation, we chose $V_{b R}=\{0.015,0.020,0.025,0.030,0.035\}$ and $V_{b T}=$ $\{0.05,0.10,0.15,0.20,0.25\}$ to be the values we computed the RT and TT data for.

We created the IF $C_{b}$ by fitting with a gamma variate function [9] a set of real measurements acquired from a healthy mouse in a very controlled experiment. We recall that the IF is not directly involved in the numerical reduction except for its asymptotic values, but in this framework the whole IF was needed for the construction of the reference and target tissue synthetic data. The reference tissue concentration was obtained solving the differential equation (1) in the unknown $C_{R}$ and then computing equation (2) for $\mathscr{C}_{R}$ with the given $V_{b R}$. Next, equation (13) was solved for $\mathbf{C}$. The total target tissue concentration $\mathscr{C}_{T}$ was computed by equation (18) with the given $V_{b T}$. Finally, the data were affected by white Gaussian noise with a signal-to-noise ratio of $30 \mathrm{~dB}$ to produce realistic signals for the activity of the radio-tracer in tissues.

The synthetic data for $\mathscr{C}_{R}$ and $\mathscr{C}_{T}$ were used to estimate the RTM parameters. The angular coefficient $\beta_{1}$ was obtained from the asymptotic values of $\mathscr{C}_{R}$ and $C_{b}$ by means of equation (6), where $t_{0}$ was identified with a time point where the concentrations started being almost constant. Consequently, by equation (5) with the selected value for $V_{b R}$, an estimate of $\lambda$ was obtained, and inserted into the subsequent inversion procedure.
The regularized $\mathrm{G}-\mathrm{N}$ scheme was applied to synthetic data in order to reconstruct the exchange coefficients $k_{1 R}, k_{1}, k_{2}$, $k_{3}, k_{4}$. The starting point of the method was randomly chosen in the interval $(0,1)$. To stop the iterative algorithm we checked the discrepancy between the reconstructed concentration and the original noisy one (the datum in the real data context), using a threshold $\left(10^{-2}\right)$ as a stopping criterion. The regularization parameter was optimized at each iteration through the Generalized Cross Validation (GCV) [10] method, by the requirement of a predefined range of variability (between $10^{4}$ and $\left.10^{6}\right)$.

The full compartmental model for TT, with known $C_{b}$, and the standard RTM, have been built similarly, and the kinetic coefficients estimated by means of the state-of-theart L-N algorithm. For the standard RTM, we have generated the synthetic data without assumption of equal volumes of distribution, and reconstructed the coefficients by imposing the condition $k_{1} / k_{2}=\epsilon k_{1 R} / k_{2 R}$, with $\epsilon \in U_{1}$ (a neighbourhood of 1). The case $\epsilon=1$ obviously corresponds to the standard RTM assumption, $\epsilon<1$ to some weaker standard RTM assumption and $\epsilon>1$ to some stronger one, testing the limit of sensitivity of the model.

In Table $\mathrm{I}$ and Table $\mathrm{II}$ results are shown concerning the sensitivity of the proposed RTM and the standard RTM. The proposed model results very stable with respect to misestimation (up to a $40 \%$ ) of both the volume fractions. The standard RTM exhibits a lower robustness and stability. In this case, data have been built imposing the condition $\epsilon=0.6$, and reconstructing with varying $\epsilon$. We can observe that the reconstruction fails for $\epsilon=1$, meaning that the standard RTM assumptions is not reliable when there is a $40 \%$ misestimation. For weaker standard RTM conditions (corresponding to $\epsilon<1$ values) the reconstructions gradually approach the true values, while going beyond (i.e. for $\epsilon>1$ ) the reconstructions get worse. This result shows that the standard RTM assumption is very sensitive to the model hypothesis of equal volumes of distribution.

Table III shows the comparison between the ground-truth and the estimated values of the parameters retrieved with $\mathrm{G}-$ $\mathrm{N}$, providing information about the reliability of the inversion procedure. It is apparent that the means provide a good approximation of the ground-truth values while the standard deviations are systematically small, thus showing the notable numerical stability of the iterative reconstruction scheme with respect to noise. Moreover, the observation that the complete inversion procedure has been based on an approximate value of $\lambda$ shows that the algorithm is very robust. The proposed $\mathrm{G}-$ $\mathrm{N}$ algorithm is also clearly compatible with the state-of-theart L-M algorithm, by producing the same mean values and comparable standard deviations (see Table III). It has indeed the advantage of being faster, given that in $\mathrm{G}-\mathrm{N}$ it is possible to compute the gradient of the functional to be minimized in an analytical form, thus avoiding time-consuming numerical differentiation step. The computational burden is $\simeq 10$ seconds for one run of $\mathrm{L}-\mathrm{M}$ and $\simeq 2$ seconds for one run of $\mathrm{G}-\mathrm{N}$ (on a Intel Core i7, 3.1GHz). 


\begin{tabular}{c|c|c|c|c|c|c}
\hline & $\lambda$ & $k_{1 R}$ & $k_{1}$ & $k_{2}$ & $k_{3}$ & $k_{4}$ \\
\hline g. t. & 2 & 0.1 & 0.3 & 0.2 & 0.05 & 0.02 \\
\hline \hline proposed RTM $\left(V_{b R}=0.015 V_{b T}=0.15\right)$ & 1.917 & $0.097 \pm 0.009$ & $0.311 \pm 0.036$ & $0.208 \pm 0.033$ & $0.061 \pm 0.015$ & $0.019 \pm 0.009$ \\
\hline proposed RTM $\left(V_{b R}=0.020 V_{b T}=0.15\right)$ & 1.826 & $0.101 \pm 0.008$ & $0.316 \pm 0.028$ & $0.200 \pm 0.030$ & $0.058 \pm 0.016$ & $0.023 \pm 0.011$ \\
\hline proposed RTM $\left(V_{b R}=0.025 V_{b T}=0.15\right)$ & 1.811 & $0.099 \pm 0.009$ & $0.300 \pm 0.033$ & $0.181 \pm 0.031$ & $0.055 \pm 0.015$ & $0.023 \pm 0.010$ \\
\hline proposed RTM $\left(V_{b R}=0.030 V_{b T}=0.15\right)$ & 1.802 & $0.102 \pm 0.008$ & $0.300 \pm 0.029$ & $0.184 \pm 0.028$ & $0.055 \pm 0.016$ & $0.022 \pm 0.011$ \\
\hline proposed RTM $\left(V_{b R}=0.035 V_{b T}=0.15\right)$ & 1.793 & $0.105 \pm 0.010$ & $0.293 \pm 0.031$ & $0.187 \pm 0.033$ & $0.061 \pm 0.019$ & $0.026 \pm 0.011$ \\
\hline \hline proposed RTM $\left(V_{b R}=0.025 V_{b T}=0.05\right)$ & 1.811 & $0.106 \pm 0.007$ & $0.293 \pm 0.025$ & $0.158 \pm 0.031$ & $0.052 \pm 0.017$ & $0.021 \pm 0.011$ \\
\hline proposed RTM $\left(V_{b R}=0.025 V_{b T}=0.10\right)$ & 1.811 & $0.108 \pm 0.008$ & $0.289 \pm 0.023$ & $0.169 \pm 0.022$ & $0.058 \pm 0.015$ & $0.026 \pm 0.011$ \\
\hline proposed RTM $\left(V_{b R}=0.025 V_{b T}=0.15\right)$ & 1.811 & $0.103 \pm 0.008$ & $0.311 \pm 0.027$ & $0.193 \pm 0.027$ & $0.058 \pm 0.014$ & $0.026 \pm 0.012$ \\
\hline proposed RTM $\left(V_{b R}=0.025 V_{b T}=0.20\right)$ & 1.811 & $0.099 \pm 0.009$ & $0.329 \pm 0.038$ & $0.218 \pm 0.037$ & $0.061 \pm 0.021$ & $0.027 \pm 0.013$ \\
\hline proposed RTM $\left(V_{b R}=0.025 V_{b T}=0.25\right)$ & 1.811 & $0.096 \pm 0.008$ & $0.327 \pm 0.033$ & $0.236 \pm 0.029$ & $0.065 \pm 0.015$ & $0.029 \pm 0.008$ \\
\hline \hline full-CM & - & - & $0.301 \pm 0.009$ & $0.204 \pm 0.019$ & $0.052 \pm 0.012$ & $0.021 \pm 0.011$ \\
\hline
\end{tabular}

TABLE I

PROPOSED RTM VALIDATION EXPERIMENT: GROUND-TRUTH (G. T.) AND RECONSTRUCTED VALUES FOR THE ADIMENSIONAL PARAMETER $\lambda$ WITH A LOGAN PLOT APPROACH AND FOR THE TRACER KINETIC PARAMETERS WITH OUR REGULARIZED G-N METHOD IN THE CASE OF DATA GENERATED WITH VARYING BLOOD VOLUME FRACTIONS (FOR BOTH RT AND TT). FOR THE INVERSION PROCEDURE, $V_{b R}=0.025$ AND $V_{b T}=0.15$. THE FULL-CM MODEL WAS OPTIMIZED THROUGH THE L-MMETHOD AND $V_{b T}=0.15$ FIXED. MEANS AND STANDARD DEVIATIONS ARE COMPUTED OVER 50 DIFFERENT RUNS OF THE ALGORITHM, WITH 50 DIFFERENT RANDOM INITIALIZATION VALUES.

\begin{tabular}{c|c|c|c|c|c|c}
\hline & $\lambda$ & $k_{1 R}$ & $k_{1}$ & $k_{2}$ & $k_{3}$ & $k_{4}$ \\
\hline g. t. & 4 & 0.05 & 0.5 & 1.2 & 0.1 & 0.04 \\
\hline \hline standard RTM $(\epsilon=0.6)$ & 3.613 & $0.054 \pm 0.001$ & $0.538 \pm 0.028$ & $1.166 \pm 0.061$ & $0.102 \pm 0.005$ & $0.041 \pm 0.004$ \\
\hline standard RTM $(\epsilon=0.7)$ & 3.613 & $0.047 \pm 0.002$ & $0.478 \pm 0.031$ & $1.208 \pm 0.078$ & $0.137 \pm 0.007$ & $0.046 \pm 0.005$ \\
\hline standard RTM $(\epsilon=0.8)$ & 3.613 & $0.043 \pm 0.001$ & $0.438 \pm 0.023$ & $1.266 \pm 0.065$ & $0.165 \pm 0.008$ & $0.047 \pm 0.005$ \\
\hline standard RTM $(\epsilon=0.9)$ & 3.613 & $0.039 \pm 0.001$ & $0.414 \pm 0.026$ & $1.345 \pm 0.085$ & $0.193 \pm 0.008$ & $0.047 \pm 0.004$ \\
\hline standard RTM $(\epsilon=1.0)$ & 3.613 & $0.037 \pm 0.001$ & $0.393 \pm 0.025$ & $1.421 \pm 0.089$ & $0.220 \pm 0.011$ & $0.047 \pm 0.005$ \\
\hline standard RTM $(\epsilon=1.1)$ & 3.613 & $0.035 \pm 0.001$ & $0.383 \pm 0.026$ & $1.521 \pm 0.102$ & $0.244 \pm 0.011$ & $0.046 \pm 0.004$ \\
\hline standard RTM $(\epsilon=1.2)$ & 3.613 & $0.033 \pm 0.001$ & $0.376 \pm 0.026$ & $1.631 \pm 0.111$ & $0.269 \pm 0.009$ & $0.046 \pm 0.003$ \\
\hline standard RTM $(\epsilon=1.3)$ & 3.613 & $0.032 \pm 0.001$ & $0.369 \pm 0.024$ & $1.736 \pm 0.113$ & $0.288 \pm 0.012$ & $0.044 \pm 0.003$ \\
\hline standard RTM $(\epsilon=1.4)$ & 3.613 & $0.031 \pm 0.001$ & $0.363 \pm 0.027$ & $1.835 \pm 0.135$ & $0.311 \pm 0.015$ & $0.043 \pm 0.004$ \\
\hline \hline full-CM & - & - & $0.498 \pm 0.020$ & $1.188 \pm 0.081$ & $0.097 \pm 0.009$ & $0.038 \pm 0.005$ \\
\hline
\end{tabular}

TABLE II

STANDARD RTM VALIDATION EXPERIMENT: GROUND-TRUTH (G. T.) AND RECONSTRUCTED VALUES FOR THE ADIMENSIONAL PARAMETER $\lambda$ WITH A LOGAN PLOT APPROACH AND FOR THE TRACER KINETIC PARAMETERS WITH THE L-M METHOD IN THE CASE OF SYNTHETIC DATA GENERATED WITHOUT ASSUMPTION OF EQUAL DISTRIBUTION VOLUMES. FOR THE INVERSION PROCEDURE, THE CONDITION $k_{1} / k_{2}=\epsilon k_{1 R} / k_{2 R}$, WITH VARYING $\epsilon$, IS IMPOSED. THE FULL-CM MODEL WAS OPTIMIZED THROUGH THE L-M METHOD. MEANS AND STANDARD DEVIATIONS ARE COMPUTED OVER 50 DIFFERENT RUNS OF THE ALGORITHM, WITH 50 DIFFERENT RANDOM INITIALIZATION VALUES. HERE $V_{b R}=0.025$ AND $V_{b T}=0.15$.

\begin{tabular}{c|c|c|c|c|c|c}
\hline & $\lambda$ & $k_{1 R}$ & $k_{1}$ & $k_{2}$ & $k_{3}$ & $k_{4}$ \\
\hline g. t. & 2.5 & 0.08 & 0.1 & 0.4 & 0.2 & 0.05 \\
\hline G-N & 2.261 & $0.082 \pm 0.005$ & $0.106 \pm 0.014$ & $0.383 \pm 0.084$ & $0.211 \pm 0.044$ & $0.053 \pm 0.009$ \\
\hline L-M & 2.261 & $0.084 \pm 0.011$ & $0.121 \pm 0.061$ & $0.447 \pm 0.271$ & $0.202 \pm 0.049$ & $0.051 \pm 0.010$ \\
\hline
\end{tabular}

TABLE III

ALGORITHM VALIDATION EXPERIMENT: GROUND-TRUTH (G. T.) AND RECONSTRUCTED VALUES FOR THE ADIMENSIONAL PARAMETER $\lambda$ WITH A LOGAN PLOT APPROACH AND FOR THE TRACER KINETIC PARAMETERS WITH OUR REGULARIZED G-N METHOD AND WITH THE L-M METHOD. MEANS AND STANDARD DEVIATIONS ARE COMPUTED OVER 50 DIFFERENT RUNS OF THE ALGORITHM, WITH 50 DIFFERENT RANDOM INITIALIZATION VALUES. HERE $V_{b R}=0.025$ AND $V_{b T}=0.15$.

\section{RESULTS ON REAL DATA}

An 'Albira' micro-PET system produced by Carestream Health is currently operational at the IRCCS San Martino IST, Genova, and experiments with mice are performed by using different tracers, mainly for applications to oncology. In this section, we show the results of our reference tissue compartmental approach for the tumor environment on real experiments, concerning murine models and FDG-PET acquisition.

All animals were studied after a fasting period of six hours to ensure a steady state of substrate and hormones governing glucose metabolism. Animals were positioned on the bed of 'Albira' micro-PET system whose two-ring configuration permits to cover the whole animal body in a single bed position. A dose of 3 to $4 \mathrm{MBq}$ of FDG was injected through a tail vein, soon after the start of a dynamic list mode acquisition lasting $40 \mathrm{~min}$. The acquisition was reconstructed using the following framing rate: $10 \times 15 \mathrm{~s}+1 \times 22 \mathrm{~s}+4 \times 30 \mathrm{~s}+5 \times 60 \mathrm{~s}$ $+2 \times 150 \mathrm{~s}+5 \times 300 \mathrm{~s}$ and PET data were reconstructed using a maximum likelihood expectation maximization (MLEM) method. Animals were inoculated subcutaneously in the dorsal hip muscles with $2 \times 10^{5}$ murine cancer cell lines CT26 (colon carcinoma cell lines). In this contest, the tumor is the target tissue. More information on the sex, weight, and glycemia of the animals are reported in Table $\mathrm{V}$.

In order to obtain the experimental concentrations, each image dataset was reviewed by an experienced observer who drew three ROIs: one over the cancer lesion, one around the 
resting thigh muscle (the reference tissue used in this work), and one over the left ventricle, in order to compute the IF. We are aware that the determination of the IF is a challenging task in the case of mice. To accomplish it, for each animal model the tracer first pass was viewed in cine mode. Then a ROI was drawn in the aortic arc, in a frame where the left ventricle was particularly visible, and maintained it for all time points. The blood volume fractions were set to 0.15 and 0.025 for the target tissue and the reference tissue, respectively, according to [18] (for tumor in CT26-tumor bearing mice), [2] and [12] (for the muscle).

The experimental data obtained for a group of four mice were processed by the RTM approach proposed in this paper. Estimates of the parameters obtained for each member of the group are shown in Table IV. Means and standard deviations were computed by using 50 runs of the code for the regularized G-N algorithm, where the initialization of the kinetic parameters was performed by picking up numbers in the interval $(0,1)$ with uniform distribution and the regularization parameter was determined at each iteration through the GCV method (with a confidence interval ranging between $10^{5}$ and $10^{7}$ ). The starting time point for the graphical analysis was chosen as the time point at which the IF curve reached a plateau, that is, became approximately asymptotic (and thus stable) to the time axis.

Table IV clearly shows that the proposed RTM is able to effectively reconstruct the kinetic coefficients, with good agreement with the ones reconstructed by means of the goldstandard full compartmental model. Moreover, in Figure 2 it is possible to observe that the IF generated by the reconstructed parameters through equation (10) provides a good approximation of the experimental IF, measured on the ROI around the left ventricle, for all the mice models considered in our analysis. The red points describe the experimental IF curve, together with its standard deviation (computed according to the experimental noise level), while the black points represent the reconstructed IF curve, computed with the averaged value of the $k_{1 R}$ parameter resulting from the 50 different runs of the algorithm, together with its standard deviation. It is also possible to observe that the reconstructed IF curves tend to be smoother than the measured ones, as expectable, given that they are model-based; this effect is particularly visible when the measured IF has some oscillations (as, for instance, times $7 \mathrm{~min}-17 \mathrm{~min}$ for $\mathrm{m} 2$ ).

In general, the inter-animal variability of the estimated parameters for the target tissue is quite low, meaning that the inter-tumor variability is not significant since in all animals the inoculated cancer cell lines are of the same type. Although, the third model (m3) appears to slightly underestimate all the parameters. This effect is visible in Figure 2(c), where the comparison between the reconstructed IF and the experimental one shows a subtle underestimation of the first points, that we hypothesize is the reason for the underestimation of $k_{1}$ and consequently of the other coefficients. Finally, we observe that the second mouse model $(\mathrm{m} 2)$ appears to have a lower $\lambda$ and a higher $k_{1 R}$ with respect to the values reconstructed for the other mice models. This could be due to the quite low blood sugar levels of $\mathrm{m} 2$ (the glycaemia is $30 \mathrm{mg} / \mathrm{dl}$, see Table $\mathrm{V})$ : when glycaemia is low, the FDG is highly absorbed by the cells, due the shortage of sugar in blood. This appears to cause a higher absorption rate in the reference tissue and therefore an increase in $k_{1 R}$. This effect is not visible in the parameters concerning the target tissue kinetics, which instead are consistent with the values for the other mice. This can be considered a consequence of the fact that the tumor is a pathological tissue less affected by the environment conditions than physiological tissues.

\section{CONCLUSIONS}

Reference tissue modelling is a powerful tool in the analysis of FDG-PET data. Comparison of tracer uptake between the RT and the TT avoids explicit determination of the TAC of arterial blood. In addition, comparison of tracer kinetics in two nearby tissues is capable of reducing distortions in the interpretation of FDG uptake, which is influenced by physiological or pharmacological conditions and uptake by other organs. First, in this paper we have shown that the RTM based on a one-compartment RT and a two-compartment TT, with given non-vanishing blood volume fractions, is identifiable. Second, we have developed an algorithm for the reduction of the RTM which leads to a unique determination of the exchange parameters. Third, we have solved the inverse problem by applying a Gauss-Newton algorithm which is very efficient and robust. Fourth, we have validated our approach with application to synthetic data and we have shown that it works equally well in the case of real data.

Our approach depends on an application of the Logan plot algorithm to the RT; indeed the procedure requires the asymptotic behaviour of the tracer concentration $C_{b}$ inside the arterial blood, which however can be estimated rather reliably. The estimated value of the angular coefficient reduces to five the total number of unknown parameters.

Validation on synthetic data has shown that our approach is capable of reconstructing with sufficient accuracy the groundtruth values of the parameters. Moreover, the formulation of the problem of determining the rate coefficients as the solution of a zero finding problem, solved by application of a Gauss-Newton approach, has given better results than the approach based on multilinear fitting via the LevenbergMarquardt method, being faster in the numerical derivative computation. In addition, results on real data seem to be in agreement with recent findings in literature (see, for instance, Figure 7 and Table 1 in [13]), even if our purpose was more a model-validation one, and we did not mean to perform an in-depth study of real oncological data.

The algorithm can be applied voxel-wise, which means that dependence of the rate coefficients on position can be explicitly considered. In view of forthcoming applications to human patients we also observe that the approach can be applied when the concentrations $\mathscr{C}_{R}$ and $\mathscr{C}_{T}$ (and $C_{b}$ ) are evaluated on a time interval $\left(t_{0}, t\right)$, with $t_{0}$ placed well after the peak of the arterial blood concentration.

\section{REFERENCES}

[1] Alpert N M, Badgaiyan R D, Livni E, and Fischman A J 2003 A novel method for noninvasive detection of neuromodulatory changes in specific neurotransmitter systems. Neuroimage 19 1049-1060. 


\begin{tabular}{c|c|c|c|c|c|c|c}
\hline & & $\lambda$ & $k_{1 R}$ & $k_{1}$ & $k_{2}$ & $k_{3}$ & $k_{4}$ \\
\hline $\mathrm{m} 1$ & proposed RTM & 4.373 & $0.039 \pm 0.003$ & $0.206 \pm 0.034$ & $0.426 \pm 0.097$ & $0.099 \pm 0.022$ & $0.032 \pm 0.009$ \\
\hline $\mathrm{m} 1$ & full-CM & - & - & $0.213 \pm 0.001$ & $0.441 \pm 0.007$ & $0.101 \pm 0.003$ & $0.033 \pm 0.001$ \\
\hline \hline $\mathrm{m} 2$ & proposed RTM & 1.858 & $0.433 \pm 0.024$ & $0.292 \pm 0.033$ & $0.468 \pm 0.117$ & $0.091 \pm 0.035$ & $0.033 \pm 0.014$ \\
\hline $\mathrm{m} 2$ & full-CM & - & - & $0.229 \pm 0.004$ & $0.481 \pm 0.022$ & $0.096 \pm 0.006$ & $0.017 \pm 0.002$ \\
\hline \hline $\mathrm{m} 3$ & proposed RTM & 5.049 & $0.111 \pm 0.002$ & $0.223 \pm 0.025$ & $0.493 \pm 0.071$ & $0.069 \pm 0.019$ & $0.023 \pm 0.011$ \\
\hline $\mathrm{m} 3$ & full-CM & - & - & $0.279 \pm 0.004$ & $0.586 \pm 0.017$ & $0.112 \pm 0.004$ & $0.031 \pm 0.001$ \\
\hline \hline $\mathrm{m} 4$ & proposed RTM & 5.844 & $0.036 \pm 0.003$ & $0.238 \pm 0.029$ & $0.350 \pm 0.075$ & $0.161 \pm 0.035$ & $0.054 \pm 0.015$ \\
\hline $\mathrm{m} 4$ & full-CM & - & - & $0.231 \pm 0.003$ & $0.435 \pm 0.011$ & $0.151 \pm 0.003$ & $0.035 \pm 0.001$ \\
\hline
\end{tabular}

TABLE IV

REAL DATA VALIDATION EXPERIMENT: RECONSTRUCTED VALUES FOR THE ADIMENSIONAL PARAMETER $\lambda$ AND FOR THE TRACER KINETIC PARAMETERS OF FDG-PET EXPERIMENTS INVOLVING FOUR MICE. BOTH THE RTM AND THE FULL-CM MODELS WERE OPTIMIZED THROUGH THE REGULARIZED G-N METHOD. THE UNCERTAINTIES ARE COMPUTED AS STANDARD DEVIATIONS OVER 50 RUNS OF THE ALGORITHM WITH 50 DIFFERENT RANDOM INITIALIZATION VALUES. HERE $V_{b R}=0.025$ AND $V_{b T}=0.15$

\begin{tabular}{c|c|c|c|c|}
\hline & Cell Line Type & Weight $[\mathrm{g}]$ & Sex & Glycemia $[\mathrm{mg} / \mathrm{dl}]$ \\
\hline $\mathrm{m} 1$ & CT26 & 17.2 & F & 84 \\
\hline $\mathrm{m} 2$ & CT26 & 16.8 & $\mathrm{~F}$ & 30 \\
\hline $\mathrm{m} 3$ & CT26 & 15.9 & F & 53 \\
\hline $\mathrm{m} 4$ & CT26 & 16.1 & F & 81 \\
\hline
\end{tabular}

TABLE V

CELL LINE TYPE, WEIGHT, SEX AND GLYCEMIA INFORMATION FOR THE FOUR MICE MODELS.

[2] Bertoldo A, Peltoniemi P, Oikonen V, Knuuti J, Nuutila P and Cobelli C 2001 Kinetic modeling of [18F] FDG in skeletal muscle by PET: a four-compartment five-rate-constant model. Am. J. Physiol. Endocrinol. Metab. 281 (3) E524-E536

[3] Delbary F, Garbarino S, and Vivaldi V 2016 Compartmental analysis of dynamic nuclear medicine data: models and identifiability. Inverse Problems 32125010 (24pp).

[4] Delbary F, and Garbarino S 2016 Compartmental analysis of dynamic nuclear medicine data: regularization procedure and application to physiology ArXiv:1608.01825.

[5] Erlandsson K, Liljeroth M, Atkinson D, Arridge S, Ourselin S, and Hutton B F 2016 Improved Parameter-Estimation With MRI-Constrained PET Kinetic Modeling: A Simulation Study. IEEE Trans. on Nucl. Science

[6] Garbarino S, Caviglia G, Sambuceti G, Benvenuto F, and Piana M 2014 A novel description of FDG excretion in the renal system: application to metformin-treated models, Phys. Med. Biol. 59 2469-2484.

[7] Garbarino S, Vivaldi V, Delbary F, Caviglia G, Piana M, Marini C, Capitanio S, Calamia I, Buschiazzo A, and Sambuceti G 2015 A new compartmental method for the analysis of liver FDG kinetics in small animals, Eur. J. Nucl. Med. Mol. Imag. Research 5 35-44.

[8] Ginovart N, Wilson A A, Meyer J H, Hussey D, and Houle S 2001 Positron Emission Tomography Quantification of [11C]-DASB Binding to the Human Serotonin Transporter: Modeling Strategies J. Cer. Blood Flow Metab. 21 1342-1353.

[9] Golish S R, Hove J D, Schelbert H R, and Gambhir S S 2001 A fast nonlinear method for parametric imaging of myocardial perfusion by dynamic 13N-ammonia PET J Nuc. Med. 42 924-931.

[10] Golub G H, Heath M, and Wahba G 1979 Generalized cross-validation as a method for choosing a good ridge parameter Technometrics 21 (2) 215-223.

[11] Gunn R N, Gunn S R, and Cunningham V J 2001 Positron Emission Tomography Compartmental Models J. Cer. Blood Flow Metab. 21 635-652.

[12] Hindel S, Söhner A, Maaß M, Sauerwein W, Möllmann D, Baba H A, Kramer M, Lüdemann L 2017 Validation of Blood Volume Fraction Quantification with 3D Gradient Echo Dynamic Contrast-Enhanced Magnetic Resonance Imaging in Porcine Skeletal Muscle PloS one 12(1) e 0170841

[13] Kadrmas D J, Rust T C, and Hoffman J M 2013 Single-scan dual-tracer FLT+FDG PET tumor characterization Phys. Med. Biol. 58 429-449.

[14] Kimura Y, Naganawa M, Shidahar M, Ikoma Y, and Watabe H 2007 PET kinetic analysis - Pitfalls and a solution for the Logan plot Ann. Nucl. Med. 21 1-8.

[15] Lammertsma A A, and Hume S P 1996 Simplified Reference Tissue Model for PET Receptor Studies NeuroImage 4 153-158.

[16] Lindholm H, Johansson O, Jonsson C, and Jacobsson H 2012 The distribution of FDG at PET examinations constitutes a relative mech- anism: significant effects at activity quantification in patients with a high muscular uptake Eur. J. Nucl. Med. Mol. Imaging 39 1685-1690.

[17] Logan J 2003 A review of graphical methods for tracer studies and strategies to reduce bias Nucl. Med. Biol. 30 833-844.

[18] Montet X, Figueiredo J L, Alencar H, Ntziachristos V, Mahmood U and Weissleder R 2007 Tomographic fluorescence imaging of tumor vascular volume in mice 1 Radiology 242(3) 751-758.

[19] Schmidt K C, and Turkheimer F E 2002 Kinetic modeling in positron emission tomography Q. J. Nucl. Med. Mol. Imaging 46 70-85.

[20] Tichauer K M, Samkoe K S, Klubben W S, Hasan T, and Pogue B W 2012a Advantages of a dual-tracer model over reference tissue models for binding potential measurement in tumors Phys Med Biol 576647 6659.

[21] Tichauer K M, Samkoe K S, Sexton K J, Hextrum S K, Yang H H, Klubben W S, Gunn J R, Hasan T, and Pogue B W 2012b In Vivo Quantification of Tumor Receptor Binding Potential with DualReporter Molecular Imaging Mol. Imaging Biol. 14 584-592.

[22] Tichauer K M, Diop M, Elliott J T, Samkoe K S, Hasan T, St. Lawrence K, and Pogue B W 2014 Accounting for pharmacokinetic differences in dual-tracer receptor density imaging Phys. Med. Biol. 59 2341-2351.

[23] Tomasi G, Kimberley S, Rosso L, Aboagye E, and Turkheimer F 2012 Double-input compartmental modeling and spectral analysis for the quantification of positron emission tomography data in oncology Phys. Med. Biol. 57 1889-1906.

[24] Watabe H, Ikoma Y, Kimura Y, Naganawa M, and Shidahara M, 2006 PET kinetic analysis - compartmental model Ann. Nucl. Med. 20583 588.

[25] Zanotti-Fregonara P, Chen K, Liow J, Fujita M, and Innis R B 2013 Image-derived input function for brain PET studies: many challenges and few opportunities J. Cer. Blood Flow Metab. 31 1986-1998.

[26] Zhou Y, Endres C J, Brasic J R, Huang S, and Wong D S 2003 Linear regression with spatial constraint to generate parametric images of ligand-receptor dynamic PET studies with a simplified reference tissue model NeuroImage 18 975-989.

[27] Zhou Y, Resnick S M, Ye W, Fan H, Holt D P, Klunk W E, Mathis C A, Dannals R, and Wonga D F 2007 Using a reference tissue model with spatial constraint to quantify [11C]Pittsburgh compound B PET for early diagnosis of Alzheimer's disease NeuroImage 36 298-312. 


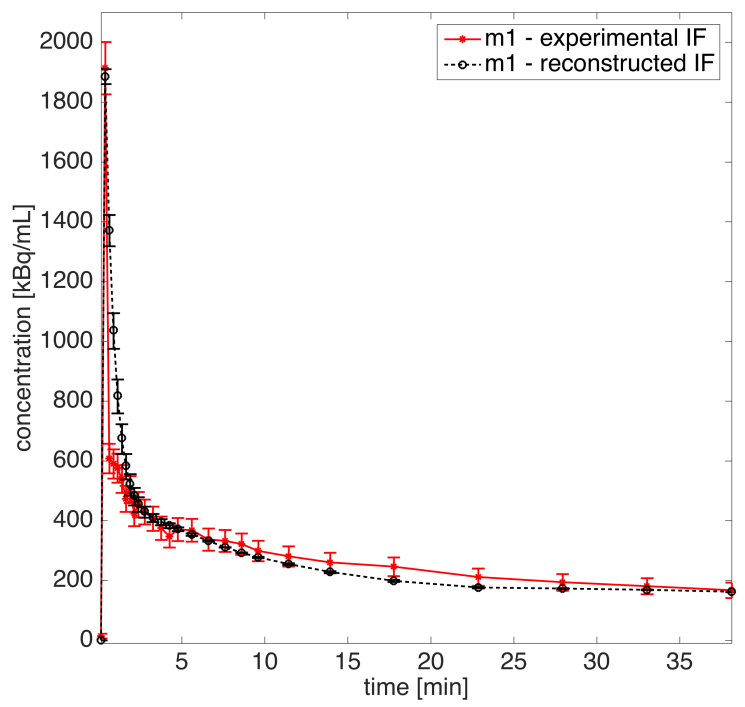

(a) $\mathrm{m} 1$

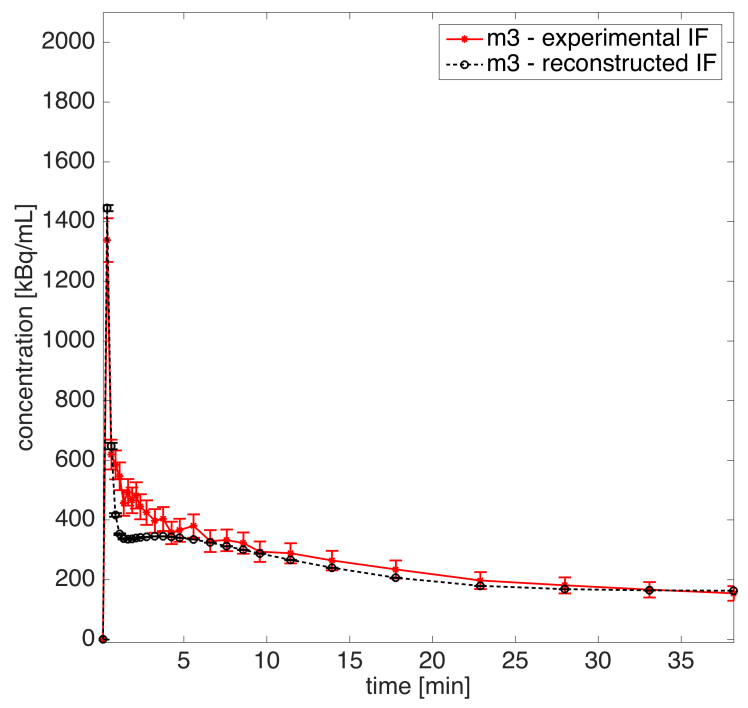

(c) $\mathrm{m} 3$

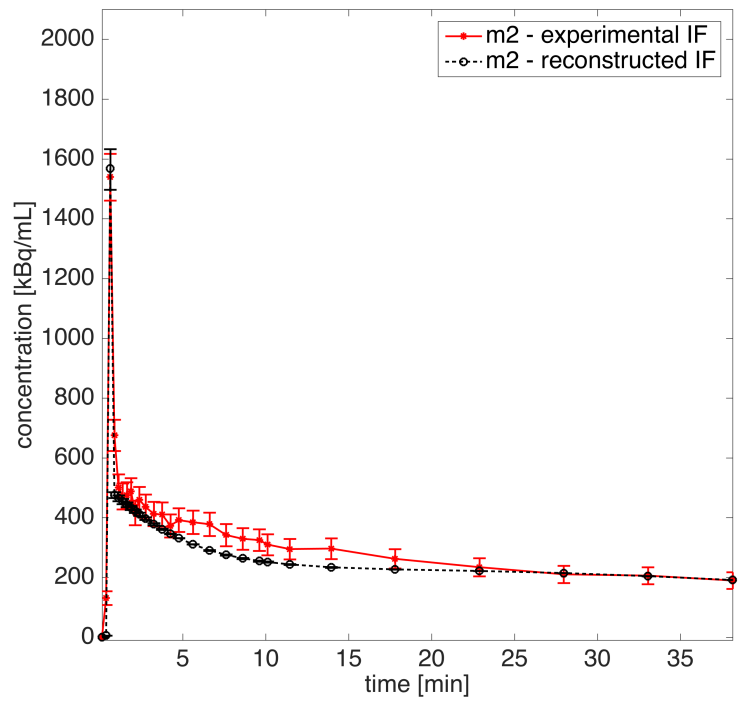

(b) $\mathrm{m} 2$

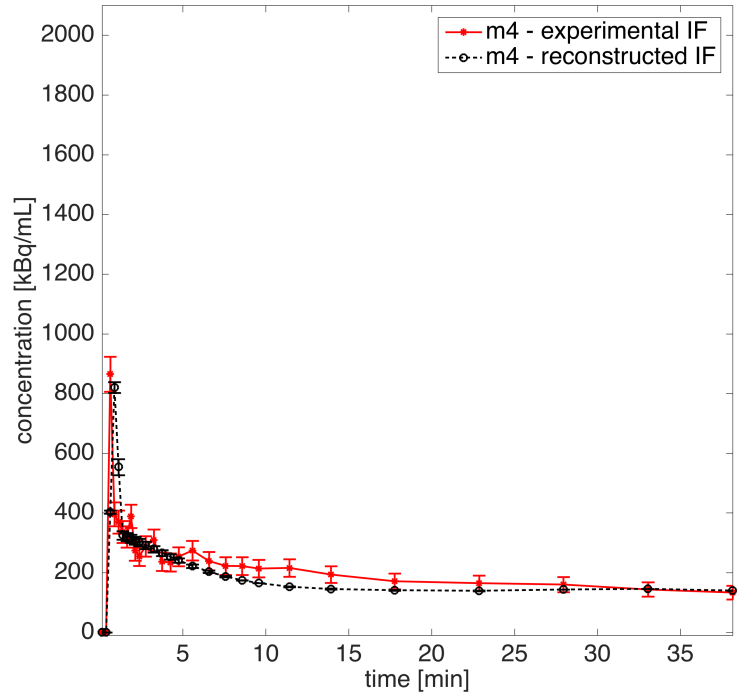

(d) $\mathrm{m} 4$

Fig. 2. Experimental IF (red curve) together with reconstructed IF (black curve) and their standard deviations, for the four mice models analyzed in this paper. 